\title{
Prevalência de cervicalgia em estudantes universitários: uma revisão integrativa
}

\author{
Prevalence of neck pain in university students: an integrative review \\ Prevalencia del dolor de cuello en estudiantes universitarios: una revisión integradora
}

Recebido: 17/10/2021 | Revisado: 21/10/2021 | Aceito: 22/10/2021 | Publicado: 24/10/2021

\author{
Ana Karoline Correa Sousa \\ ORCID: https://orcid.org/0000-0001-6163-008X \\ Centro Universitário da Amazônia, Brasil \\ E-mail: karol.sousa10@hotmail.com \\ Dhuly Ramos da Silva \\ ORCID: https://orcid.org/0000-0003-0306-5689 \\ Centro Universitário da Amazônia, Brasil \\ E-mail: ramosdhuly@gmail.com \\ Diego Sarmento de Sousa \\ ORCID: https://orcid.org/0000-0001-5637-5985 \\ Universidade do Estado do Pará, Brasil \\ E-mail: fisioterapia.santarem@unama.br
}

\begin{abstract}
Resumo
Introdução: A cervicalgia é considerada uma afecção bastante comum atualmente. Afeta cerca de $50 \%$ de toda a população, com predominância no sexo feminino. No contexto acadêmico, o acumulo de tarefas exigidas, o tempo destinados aos estudos e a postura em flexão cervical relacionada ao uso de smartphones e computadores, aumentam a probabilidade de ocorrer disfunções musculoesquelética na região cervical. Objetivo: Conhecer a prevalência de cervicalgia em estudantes universitários e seus fatores associados. Metodologia: as bases de dados escolhidas para a realização desta pesquisa foram: PubMed, Science Direct, e Google acadêmico. Foram incluídos artigos publicados no período de 2017 a 2021, nos idiomas português e inglês, cujo título, descritores ou resumo fizessem referência a temática. Como critérios de exclusão: artigos que não encontrados na íntegra, revisão simples de literatura e aqueles que não respondessem a proposta do estudo. Resultados e Discussão: Dentre os períodos pesquisados seis são internacionais e quatro nacionais, o que representa escassez de estudos brasileiros acerca dessa temática. A maioria dos dados constataram prevalência significante de cervicalgia em estudantes universitários. Conclusão: Subsiste uma prevalência relevante de cervicalgia em estudantes universitários que tem afetado diretamente à qualidade de vida.
\end{abstract}

Palavras-chave: Prevalência; Cervicalgia; Estudantes.

\begin{abstract}
Introduction: Neck pain is currently considered a very common condition. It affects about $50 \%$ of the entire population, with a predominance of females. In the academic context, the accumulation of required tasks, the time allocated to studies and the posture in cervical flexion related to the use of smartphones and computers, increase the probability of musculoskeletal disorders in the cervical region. Objective: To know the prevalence of neck pain in university students and its associated factors. Methodology: the databases chosen for this research were: PubMed, Science Direct, and Academic Google. Articles published from 2017 to 2021, in Portuguese and English, whose title, descriptors or abstract made reference to the theme were included. As exclusion criteria: articles that were not found in full, simple literature review and those that did not respond to the study proposal. Results and Discussion: Among the periods surveyed, six are international and four are national, which represents a shortage of Brazilian studies on this topic. Most data found a significant prevalence of neck pain in university students. Conclusion: There remains a relevant prevalence of neck pain in university students, which has directly affected their quality of life.
\end{abstract}

Keywords: Prevalence; Neck pain; Students.

\section{Resumen}

Introducción: En la actualidad, el dolor de cuello se considera una afección muy común. Afecta aproximadamente al $50 \%$ de la población total, con predominio del sexo femenino. En el contexto académico, la acumulación de tareas requeridas, el tiempo destinado a los estudios y la postura en flexión cervical relacionada con el uso de teléfonos inteligentes y computadoras, aumentan la probabilidad de trastornos musculoesqueléticos en la región cervical. Objetivo: Conocer la prevalencia de dolor de cuello en estudiantes universitarios y sus factores asociados. Metodología: las bases de datos elegidas para esta investigación fueron: PubMed, Science Direct y Academic Google. Se incluyeron artículos publicados de 2017 a 2021, en portugués e inglés, cuyo título, descriptores o resumen hicieran referencia al tema. Como criterios de exclusión: artículos que no se encontraron en revisión completa de la literatura, simple y aquellos que no respondieron a la propuesta de estudio. Resultados y Discusión: Entre los períodos 
investigados, seis son internacionales y cuatro son nacionales, lo que representa una escasez de estudios brasileños sobre este tema. La mayoría de los datos encontraron una prevalencia significativa de dolor de cuello en estudiantes universitarios. Conclusión: persiste una prevalencia relevante de dolor de cuello en estudiantes universitarios, que ha afectado directamente su calidad de vida.

Palabras clave: Prevalencia; Dolor de cuello; Estudiantes.

\section{Introdução}

A cervicalgia é uma das condições dolorosas mais prevalentes nas práticas clínicas médicas a nível mundial. Ela caracteriza-se por episódios de dor e redução da amplitude de movimento de toda região cervical. Sua incidência acarreta inúmeros danos na qualidade de vida do indivíduo, visto que ultrapassa o acometimento pessoal e acaba afetando a família, economia e sistema de saúde. (Safiri et al., 2020).

A coluna cervical é construída a partir da sobreposição de sete vértebras (C1-C7), ligamentos e músculos que permitem a funcionalidade dessa estrutura: proteção, sustentação e movimentação. Por permitir ampla angulações ela se torna mais suscetível a estresses biomecânicos que podem gerar fatores que aceleram os processos degenerativos das vertebras como: cervicalgias agudas, inflamação, alterações tônicas e redução na amplitude de movimento, assim como desencadear mudanças estruturais na coluna. A apresentação da cervicalgia, difere de acordo com a estrutura motivadora, influências posturais e emocionais. Pode apresentar-se aguda, porém a mais relatada é a dor crônica agudizada (Teixeira et al., 2001). As principais causas biomecânicas se atrelam ao trauma, movimentos bruscos, esforços físicos e postura mantida prolongada que, associados à utilização de aparelhos eletrônicos e o sedentarismo, pode sugerir o aumento das chances de ocorrência em universitários nos dias atuais (Silva et al., 2012).

Em países desenvolvidos e em desenvolvimento, a dor no pescoço foi relatada coletivamente em estudantes universitários. Na Finlândia, a prevalência de dor cervical foi de 29\% (Oksanen et al., 2014), 34,6\% na Austrália (Smith \& Leggat, 2004), 54\% nos EUA (Jenkins et al., 2007), 49,7\% no Brasil (Mattos et al., 2009), 46\% na Tailândia (Kanchanomai et al., 2011), 54\% na Arábia Saudita (Gharib \& Hamid, 2013), 55,8\% na Coréia (Kim \& Kim, 2015) e 49,9\% na China (Woo et al., 2016). A associação de fatores físicos, psicossociais e fatores individuais se encaixam na literatura, como principais fatores desencadeantes na população em geral (Kanchanomai, et al., 2011). Desse modo, nota-se a importância de haver mais estudos de revisões sistemáticas para analisar esses fatores preditivos em um público específico como o de jovens universitários, visto que o crescente aparecimento e persistência dessa disfunção, pode gerar sérios problemas a posteriori.

A construção desse estudo parte do interesse em identificar essa prevalência em uma população exata e conhecer os principais fatores desencadeantes por meio de um levantamento literário científico. Dessa forma, direcionar a atuação de profissionais na prevenção dessa síndrome clínica que afeta grande parte da população jovem mundial.

\section{Metodologia}

Trata-se de um estudo de revisão integrativa da literatura, norteado pelas diretrizes do protocolo PRISMA, desenvolvido com o objetivo de substanciar achados de estudos realizados, por meio de métodos diferentes, com intuito de contribuir para a melhora do relato de revisões sistemáticas e meta-analises e aperfeiçoar o conhecimento alusivo ao tema proposto. As etapas para a construção da revisão foram: levantamento da questão da pesquisa ou definição do problema a ser pesquisado, estabelecimento de critérios de inclusão ou exclusão dos estudos, com síntese dos principais achados e análise detalhada, interpretação dos resultados com a identificação das conclusões resultantes da revisão integrativa (Moher et al., 2015).

A pergunta norteadora dessa revisão foi: Qual a prevalência de cervicalgia em universitários e quais fatores podem estar relacionados à ocorrência dessa problemática? As bases de dados bibliográficos utilizadas para a realização da pesquisa 
foram: Google Acadêmico, Pubmed e ScienceDirect.

Os critérios de inclusão foram os estudos publicados nas bases de dados, no período de 2017 a 2021, nos idiomas inglês e português, cujo título, descritores e/ou resumos fizessem referência à temática. Como critérios de exclusão determinou-se os artigos não encontrados na íntegra, revisões de literatura, publicação anteriores ao ano de 2017 e aqueles que não respondiam às questões norteadoras proposta por este estudo. Dessa forma, 10 artigos foram selecionados nas bases de dados de forma criteriosa apresentando importância no campo científico e fidelidade com o tema proposto.

Figura 1 - Fluxograma de identificação, seleção e inclusão dos estudos.

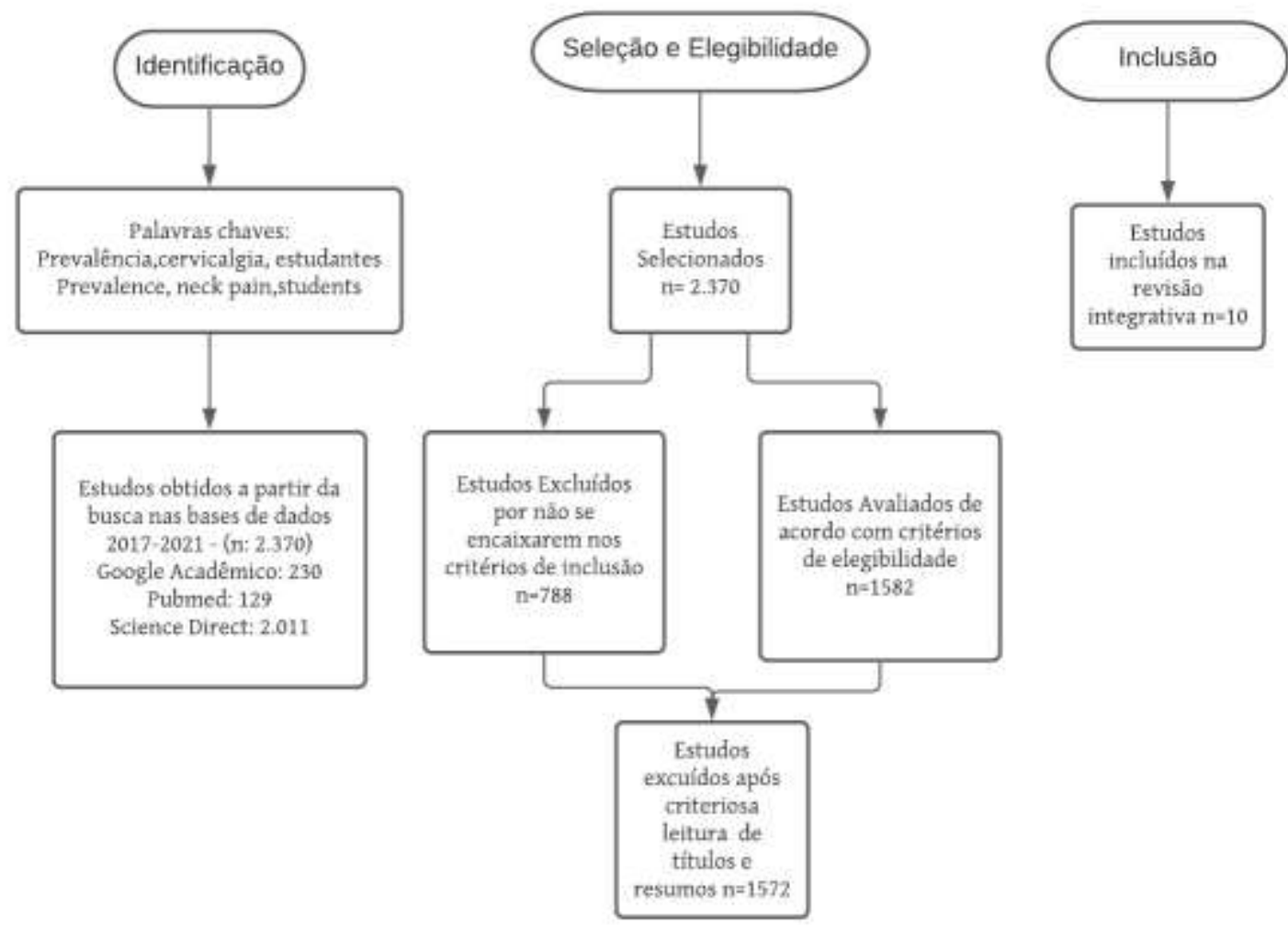

Fonte: Sousa, Silva e Sousa (2021).

\section{Resultados e Discussão}

Para melhor apresentar os resultados, foi elaborado o Quadro 1 com subcategorias que sintetizam a caracterização do estudo. 
Quadro 1 - Caracterização dos Estudos.

\begin{tabular}{|c|c|c|c|c|}
\hline AUTOR/DATA LOCAL & OBJETIVO & AMOSTRA & $\begin{array}{l}\text { MÉTODO DE } \\
\text { AVALIAÇÃO DA } \\
\text { CERVICALGIA }\end{array}$ & RESULTADOS \\
\hline $\begin{array}{c}\text { NAMWONGSA et } \\
\text { al., (2018) } \\
\text { Tailândia }\end{array}$ & \begin{tabular}{|c} 
Investigar distúrbios \\
musculoesqueléticos em \\
usuários de smartphones \\
na Tailândia, a fim de \\
confirmar a alta \\
prevalência de dor no \\
pescoço e os fatores \\
associados aos distúrbios \\
no pescoço em usuários \\
de smartphones.
\end{tabular} & $\begin{array}{l}779 \text { universitários } \\
\text { de forma aleatória } \\
\text { em } 17 \\
\text { univerisades } \\
\text { sendo } 47 \\
\text { acadêmicos de } \\
\text { cada. }\end{array}$ & $\begin{array}{l}3 \text { questionários: auto- } \\
\text { aplicável, Suanprung } \\
\text { Stress Test-20, The } \\
\text { Standardized Nordic } \\
\text { Questionnaire (SNQ). }\end{array}$ & $\begin{array}{l}\text { A prevalência de dor no pescoço foi de } \\
32,50 \% \text {. Constatou-se que os fatores } \\
\text { individuais, tabagismo e a postura de } \\
\text { flexão do pescoço estiveram associados } \\
\text { à ocorrência de distúrbios } \\
\text { musculoesqueléticos do pescoço em } \\
\text { usuários de smartphones. }\end{array}$ \\
\hline $\begin{array}{l}\text { SATO et al., (2019) } \\
\text { Brasil - São Paulo }\end{array}$ & $\begin{array}{c}\text { Conhecer a prevalência } \\
\text { dessa afecção nos alunos } \\
\text { de medicina e o } \\
\text { respectivo perfil. }\end{array}$ & $\begin{array}{l}100 \text { estudantes de } \\
\text { medicina de } \\
\text { forma aleatória } \\
\text { com idade média } \\
\text { de } 17 \text { a } 26 \text { anos. }\end{array}$ & $\begin{array}{l}2 \text { questionários: NDI( } \\
\text { Neck Disability Index) } \\
\text { e SF-36 (Medical } \\
\text { Outcome Study Short } \\
\text { Form } 36 \text { Survey). }\end{array}$ & $\begin{array}{l}\text { A prevalência de cervicalgia foi de } 34 \% \\
\text { com predomínio feminino. Dentre os } \\
\text { fatores desecandeantes a posição sentada } \\
\text { com pescoço fletido é frequentemente } \\
\text { assumida. Houve impacto na qualidade } \\
\text { de vida nos domínios aspcetos físicos, } \\
\text { dor e vitalidade. }\end{array}$ \\
\hline $\begin{array}{l}\text { ALSALAMEH et al., } \\
\text { (2019) } \\
\text { Israel - Qasin }\end{array}$ & \begin{tabular}{|} 
Determinar a prevalência \\
de dependência/uso \\
excessivo de \\
smartphones entre \\
estudantes de medicina e \\
investigar se há \\
associação entre \\
dependência de \\
smartphone e dor \\
musculoesquéletica. \\
\end{tabular} & $\begin{array}{l}242 \text { acadêmicos } \\
\text { de medicina } \\
\text { escolhidos de } \\
\text { forma aleatória } \\
\text { com idade média } \\
\text { de } 19 \text { a } 32 \text { anos }\end{array}$ & $\begin{array}{c}2 \\
\text { questionários:Addiction } \\
\text { Scale Short Version } \\
\text { (SAS-SV) e } \\
\text { musculoesquéletico } \\
\text { nórdico (NMQ). }\end{array}$ & $\begin{array}{l}\text { A prevalência de cervicalgia foi de } \\
59,5 \% \text {, e o principal fator associado foi o } \\
\text { uso prolongado de smartphones que teve } \\
\text { uma relação significativa com as dores } \\
\text { musculoesqueéticas. }\end{array}$ \\
\hline $\begin{array}{l}\text { Da SILVA et al., } \\
\text { (2020) } \\
\text { Brasil - Maranhão }\end{array}$ & $\begin{array}{l}\text { Avaliar a qualidade de } \\
\text { vida e a prevalência de } \\
\text { dor na região cervical } \\
\text { em acadêmicos. }\end{array}$ & $\begin{array}{c}289 \text { acadêmicos } \\
\text { de Fisioterapia, } \\
\text { Engenharia Civil, } \\
\text { Arquitetura, } \\
\text { Educação física e } \\
\text { Análise de } \\
\text { Desenvolviment } \\
\text { o de sistema; } \\
\text { idade média: } 18 \\
\text { a } 50 \text { anos, de } \\
\text { ambos os sexos. }\end{array}$ & $\begin{array}{l}2 \text { questionários: SF36 e } \\
\text { Beckpei. }\end{array}$ & $\begin{array}{l}\text { A prevalência de cervicalgia foi de } 30 \% \\
\text { com predominio do sexo feminino. Os } \\
\text { fatores associados foram: não praticar } \\
\text { exercícios físicos, permanência sentado } \\
\text { por tempo prolongado com uso do } \\
\text { computador e assitindo televisão. }\end{array}$ \\
\hline $\begin{array}{c}\text { Tourinho \& Junior } \\
\text { (2020) } \\
\text { Brasil - Bahia }\end{array}$ & $\begin{array}{l}\text { Indentificar a } \\
\text { cervicalgia Inespecífica } \\
\text { em estudantes de } \\
\text { fisioterapia de uma } \\
\text { instituição privada. }\end{array}$ & $\begin{array}{l}30 \text { acadêmicos } \\
\text { de fisioterapia de } \\
\text { ambos os sexos . } \\
\text { Com idade } \\
\text { média de } 18 \text { a } 60 \\
\text { anos. }\end{array}$ & $\begin{array}{c}3 \text { questionários: } \\
\text { Questionário de escala } \\
\text { funcional de } \\
\text { incapacidade do } \\
\text { pescoço de } \\
\text { Copenhagem, Escava } \\
\text { visual analógica de } \\
\text { dor (EVA) e } \\
\text { questionário } \\
\text { sociodemogáfico. } \\
\end{array}$ & $\begin{array}{l}\text { A prevalência de cervicalgia foi de } \\
63,3 \% \text { na amostra, idade média foi de } \\
24,67 \% \text { com predominância o sexo } \\
\text { feminino. O trabalho repetitivo, } \\
\text { estresse, traumatismos prévios no } \\
\text { pescoço e ombros, esforço e a postura } \\
\text { por longo períodos foram os principais } \\
\text { desencadeantes para a cervicalgia. }\end{array}$ \\
\hline $\begin{array}{l}\text { CHAN et al., (2020) } \\
\text { China - Hong Kong }\end{array}$ & $\begin{array}{l}\text { Comparar a prevalência } \\
\text { de cervicalgia entre } \\
\text { estudantes de diferentes } \\
\text { programas de graduação } \\
\text { e investigar seus fatores } \\
\text { de risco. }\end{array}$ & $\begin{array}{l}1.002 \\
\text { acadêmicos de } \\
\text { Fisioterapia, } \\
\text { Enfermagem e } \\
\text { Administração } \\
\text { selecionados de } \\
\text { forma aleatória. }\end{array}$ & $\begin{array}{l}\text { Pesquisa online } \\
\text { autoaplicável. }\end{array}$ & $\begin{array}{l}\text { O estudo demostrou uma prevalência de } \\
22,3 \% \text {. Ansiedade, altura e uso } \\
\text { prolongado de smartphone foram } \\
\text { significamente associados à presenca de } \\
\text { dor cervical. }\end{array}$ \\
\hline
\end{tabular}




\begin{tabular}{|c|c|c|c|c|}
\hline $\begin{array}{c}\text { BEHERA et al., (2020) } \\
\text { Índia Central }\end{array}$ & $\begin{array}{c}\text { Avaliar a dor no pescoço } \\
\text { e os fatores associados, } \\
\text { incluíndo o uso de } \\
\text { dispositivos entre } \\
\text { estudantes de medicina } \\
\text { de primeira linha na } \\
\text { Índia central. } \\
\end{array}$ & $\begin{array}{l}331 \text { acadêmicos } \\
\text { de medicina } \\
\text { selecionados } \\
\text { aleatóriamente. } \\
\text { Idade média de } \\
20 \text { anos. }\end{array}$ & $\begin{array}{l}\text { Questionário } \\
\text { autoaplicável. }\end{array}$ & $\begin{array}{l}\text { A prevalência de cervicalgia foi } \\
58,3 \% \text {. O agravamento autopercebido } \\
\text { da dor, o uso de dispositivos } \\
\text { eletrônicos teve chances } \\
\text { significativamente maiores de } \\
\text { contribuir para o episódio de dor } \\
\text { cervical. } \\
\end{array}$ \\
\hline $\begin{array}{l}\text { KUC et al., (2020) } \\
\text { Polônia - Bialystok }\end{array}$ & $\begin{array}{c}\text { Avaliar a prevalência de } \\
\text { disfunção da coluna } \\
\text { cervical entre estudantes } \\
\text { de odontologia de uma } \\
\text { faculdade de medicina. }\end{array}$ & $\begin{array}{c}112 \text { acadêmicos } \\
\text { de Odontologia } \\
\text { (73 mulheres e } 39 \\
\text { homens) com } \\
\text { idade média de } 20 \\
\text { a } 32 \text { anos. } \\
\end{array}$ & $\begin{array}{l}\text { Questionário } \\
\text { autoaplicável }\end{array}$ & $\begin{array}{l}\text { A prevalência de dor cervical foi de } \\
\text { 47,32\% . Os fatores associados: } \\
\text { estresse,deficiência cervical, } \\
\text { distubrios da coluna vertebral, } \\
\text { sobrecarga, predisposição individual e } \\
\text { perfil biopsciossocial, se destacaram. }\end{array}$ \\
\hline $\begin{array}{l}\text { OLIVEIRA } \\
\text { et al., (2021) } \\
\text { Brasil - Piauí }\end{array}$ & $\begin{array}{l}\text { Analisar a prevalência } \\
\text { de cervicalgia em } \\
\text { alunos de fisioterapia } \\
\text { de uma Faculdade de } \\
\text { teresina que fazem uso } \\
\text { prolongado de } \\
\text { smartphones. }\end{array}$ & $\begin{array}{l}41 \text { acadêmicos } \\
\text { de ambos os os } \\
\text { sexo. Com idade } \\
\text { média de } 22 \text { a } \\
\quad 46 \text { anos. }\end{array}$ & $\begin{array}{l}2 \text { Questionários: } \\
\text { questionário de } \\
\text { perguntas fechadas e } \\
\text { Maastricht Upper } \\
\text { Extremity } \\
\text { Questionnarie } \\
\text { (MUEQ-BR). }\end{array}$ & $\begin{array}{c}\text { A idade média foi de } 27,5 \text { anos com } \\
\text { predominância no sexo feminino. } \\
\text { Não houve uma maior prevalência } \\
\text { de cervigalgia e de dor na região da } \\
\text { face, têmporas e região do ouvido } \\
\text { quando comparado o tempo de uso } \\
\text { de smartphone. }\end{array}$ \\
\hline $\begin{array}{c}\text { AYHUALEN et al., } \\
(2021) \\
\text { Etiópia - Gondar }\end{array}$ & $\begin{array}{l}\text { Indentificar a prevalência } \\
\text { e os fatores associados à } \\
\text { dor cervical em usuários } \\
\text { de smartphones da } \\
\text { Universidadede de } \\
\text { Gondar. }\end{array}$ & $\begin{array}{l}845 \text { acadêmicos } \\
\text { escolhidos de } \\
\text { forma aleatória. } \\
\text { Idade média de } \\
21,9 \text { anos. }\end{array}$ & $\begin{array}{c}\text { Questionário } \\
\text { autoaplicável adaptado } \\
\text { do questionário } \\
\text { musculoesquelético } \\
\text { nórdico. }\end{array}$ & $\begin{array}{l}\text { A prevalência de dor cervical foi de } \\
47,4 \% \text {, entre os fatores } \\
\text { desencadeantes, frequentar a } \\
\text { universidade no último ano, } \\
\text { características pessoais, uso de } \\
\text { smartphones por longo período, jogar } \\
\text { games, maior tempo de uso de mídia } \\
\text { social, foram os principais. }\end{array}$ \\
\hline
\end{tabular}

Fonte: Sousa, Silva e Sousa (2021).

De forma criteriosa, foram selecionados 10 artigos, 4 nacionais e 6 internacionais, entre os anos de 2018 e 2021 . Nos estudos acima estão contidos a epidemiologia da cervicalgia e os fatores associados, com o número amostral que variaram entre 30 a 1002 indivíduos. A caracterização da amostra deu-se por estudantes de Medicina, Fisioterapia, Engenharia Civil, Arquitetura, Educação Física, Análise de Desenvolvimento de Sistema, Enfermagem, Odontologia e alunos escolhidos de forma aleatória, com idades que variaram entre 17 a 60 anos. Os questionários utilizados como forma metodológica foram: questionário Autoaplicável, Escala Visual Analógica de dor (EVA), Escala Funcional de Incapacidade do Pescoço de Copenhagem, Sóciodemográfico, Musculoesquético Nórdico, Suanprung Stress Test-20, NDI (Neck Disability Index), SF-36 (Medical Outcome Study Short Form 36 Survey), Addiction Scale Short Version (SAS-SV), Beckpei e Maastricht Upper Extremity Questionnarie (MUEQ-BR). Os resultados apontaram considerável prevalência de cervicalgia com porcentagem de $22,3 \%$ a $63,3 \%$. Os fatores encontrados foram fatores individuais, tabagismo, postura de flexão do pescoço, uso prolongado de smartphones, sedentarismo, permanência na postura sentada por tempo excessivo, assistir televisão, trabalhos repetitivos, estresse, traumatismos prévios no pescoço e ombro, esforço físico, frequentar a faculdade no último ano, jogar vídeo games, tempo de uso das mídias sociais, ansiedade, deficiência cervical, distúrbios na coluna vertebral e sobrecarga.

De acordo com Namwongsa et al., (2018) o uso prolongado de smartphones associados com a postura em flexão do pescoço mostrou-se como um dos principais distúrbios musculoesqueléticos cervicais dos acadêmicos da Tailândia, com o percentual de 32,50\%. Corroborando com essa temática, os autores Alsalameh et al., 2019, Ayhualen et al., 2021 e Chan et al., (2020) em seus estudos apresentaram respectivamente percentuais de prevalência de 59,5\%, 47,4\%, 22,3\% associada ao uso de smartphones, contribuindo significativamente para a apresentação de dor cervical. Seguindo a mesma linha de raciocínio, Delfino et al., (2012) em seus dados estatísticos afirma que a prevalência de cervicalgia na população em geral varia de $10 \%$ a $15 \%$, acometendo em torno de $67 \%$ a $70 \%$ dos indivíduos em algum momento de sua vida. Como fatores predisponentes cita o uso de computadores, smartphones e postura inadequada. No estudo de Vittar et al., (2021), a prevalência de cervicalgia em 
estudantes do ensino médio foi quantificada em 49,1\% relacionada ao uso de dispositivos eletrônicos como fator contribuinte para essa disfunção. Ao analisar os aspectos abordados, a apresentação de cervicalgia em diferentes populações mostra-se em comum dominância, onde a utilização de equipamentos eletrônicos é sugerido como principal fator de influência na configuração de dor cervical.

Em contrapartida, Oliveira et al., (2021), ao analisar a prevalência de dor cervical em alunos de fisioterapia que fazem o uso prolongado de smartphones, observou em seu resultado que não houve uma maior prevalência quando relacionado ao tempo de uso de smartphones e pescoço flexionado, porém $48,8 \%$ dos jovens da sua pesquisa utilizavam os aparelhos na postura deitada, que biomecanicamente diminui a sobrecarga na coluna cervical. A utilização de outros dispositivos eletrônicos como computador, televisão e vídeo game também são sugeridos como fatores relevantes no desenvolvimento da disfunção na região do pescoço (Behera et al., 2020; Ayhualen et al., 2021; Da Silva et al., 2020).

Em relação a questão postural, a posição sentada e cabeça flexionada foi apontada pelos autores Sato, et al., 2019 e Silva, et al., 2020 como fator preditivo que pode atenuar o estresse biomecânico e acarretar alterações nas curvaturas fisiológicas da coluna vertebral. Uma vez que segundo Mehrdad et al., (2012), a cervicalgia está diretamente relacionada a períodos prolongados da postura sentada e pescoço flexionado. Como resposta fisiológica, Lee et al., (2014) cita que a excessiva flexão do pescoço pode acarretar danos à região circundante às vértebras e ligamentos, causar a hiperativação dos músculos extensores, resultando em micro lesões na estrutura e motivando episódios cada vez mais frequentes de dor.

Segundo Neto e colaboradores (2021) \& Assis et al., (2013), durante o período de graduação, o universitário é submetido a vários fatores psicossociais que afetam diretamente à qualidade de vida, dentre esses fatores, destacam-se o estresse constante, excessivas preocupações quanto à inserção no mercado de trabalho, primeiro contato com estágios, produção de atividades acadêmicas e períodos extensos de estudo. Para Kuc et al., (2020) as falhas em relacionamentos afetivos, baixos níveis de renda financeira, ausência de conhecimentos gerais e ocorrência de outras disfunções crônicas já instaladas reforçam os estudos de Ayhualen et al., (2021) \& Tourinho \& Junior, (2020), quando relacionam os aspectos biopsicossociais com o desenvolvimento de cervicalgia.

Em consonância à condição universitária, a ausência de atividades físicas atrelada a todos os fatores acima já citados, pode está diretamente ligada a alta prevalência de algias, posto que o sedentarismo é conhecido como um potencial fator de risco para afecções na coluna vertebral (Vey, Silva \& Lima, 2013). Frente a isso essa inatividade causa enfraquecimento da musculatura adjacente deixando-a vulnerável à reações disfuncionais. Em contrapartida a frequência de atividade física melhora não só a parte orgânica, mas toda a questão psicossocial, como autoestima, bem estar físico e mental (Mancin et al., 2008).

Na pesquisa, foi identificado predomínio de cervicalgia no sexo feminino, desse modo, o estudo de Al-Hadidi et al., (2019) vem corroborando com a mesma estatística, visto que as mulheres utilizavam os aparelhos eletrônicos por mais tempo que os homens para desenvolver seus trabalhos e estudos. Na literatura, as explicações para esse fator podem ser relacionadas ao fato de as mulheres apresentarem maiores incidências de dores musculoesqueléticas e maior predisposição às dores cônicas (Fillingim, et al., 2009), extensas horas de jornada de trabalho que excedem sua capacidade física e maiores estresses emocionais atrelados a fatores hormonais (Silva et al., 2017).

\section{Conclusão}

De acordo com os resultados e seguindo os nossos critérios de seleção dos manuscritos, notamos poucos estudos relevantes sobre a prevalência de cervicalgia em estudantes universitários, limitando nossas observações em 10 artigos.

Evidenciamos que a prevalência de dores na região cervical em estudantes universitários variou de 22,3\% a 63,3\%, sendo que esses resultados estavam associados com sexo feminino, o tempo prolongado de uso de dispositivos eletrônicos, 
postura sentada associado ao pescoço flexionado por logos períodos, além dos fatores psicossociais, predisposição genética e sedentarismo.

Incentivamos a realização de novos estudos sobre o tema, visto que, o assunto abordado é de grande relevância para a comunidade científica, profissional e acadêmica. Sugere-se a produção de conhecimento por meio de artigos, estudos com metodologias mais criteriosas e com uma amostragem representativa dessa população para melhor entendermos o fenômeno da cervicalgia em universitários.

\section{Agradecimentos}

Primeiramente, agradecemos a Deus pela vida, sustento e vigor para concluir a graduação. Aos nossos pais que foram nosso alicerce e que não mediram esforços para que esse nosso sonho fosse realizado. Ao nosso orientador que nos deu todo amparo e repassou todo o seu conhecimento afim de nos ajudar na construção desse trabalho. A todos os nosso professores que ultrapassaram a docência e se fizeram amigos em horas precisas, todos são referências para a gente. Aos amigos que entenderam quando fomos ausentes. As pessoas especiais que nos incentivaram e nos ergueram quando o desânimo bateu à porta. Todos são responsáveis pelas profissionais que estamos nos tornando. Todos foram indispensáveis nessa caminhada e a eles, a nossa eterna gratidão.

\section{Referências}

Al-Hadidi, F., Bsisu, I., AlRyalat, S. A., Al-Zu'bi, B., Bsisu, R., Hamdan, M., Kanaan, T., Yasin, M., \& Samarah, O. (2019). Association between mobile phone use and neck pain in university students: A cross-sectional study using numeric rating scale for evaluation of neck pain. Revista PloS one. 14(5), e0217231. https://doi.org/10.1371/journal.pone.0217231

Alsalameh, A. M., Harisi, M. J., Alduayji, M. A., Almutham, A. A., \& Mahmood, F. M. (2019). Evaluating the relationship between smartphone addiction/overuse and musculoskeletal pain among medical students at Qassim University. Journal of family medicine and primary care. 8(9), 2953-2959. https://doi.org/10.4103/jfmpc.jfmpc_665_19

Assis, C. L., Silva, A. P. F., Lopes, M. S., Silva, P. C. B., \& Santini, T. O. (2013). Sintomas de Estresse em Concluintes do Curso de Psicologia de Uma Faculdade Privada do Norte do País. Revista Mudanças - Psicologia Da Saúde, 21(1). https://doi.org/10.15603/2176-1019/mud.v21n1p23-28

Ayhualem, S., Alamer, A., Dabi, S. D., Bogale, K. G., Abebe, A. B., \& Chala, M. B. (2021). Burden of neck pain and associated factors among smart phone user students in University of Gondar, Ethiopia. Revista PloS one. 16(9), 1-15. https://doi.org/10.1371/journal.pone.0256794

Behera, P., Majumdar, A., Revadi, G., Santoshi, J. A., Nagar, V., \& Mishra, N. (2020). Neck pain among undergraduate medical students in a premier institute of central India: A cross-sectional study of prevalence and associated factors. Journal of family medicine and primary care. 9(7), 3574-3581. https://doi.org/10.4103/jfmpc.jfmpc_206_20

Chan, L. L. Y., Wong, A. Y. L., Phil, M., Wang, M. H., Cheung, K., Samartzis, D. (2020). The prevalence of neck pain and associated risk factors among undergraduate students: A large-scale cross-sectional study. International Journal of Industrial Ergonomics. 56, 1-9.

Da Silva, T., Oliveira, A. L., dos Santos, S. M., Oliveira, T. de J., Guimarães, L. S., Ferreira, A. M. R., Ramos, S. D. do N., Silva, R. T. B., Cruz, T. T. da, Rodrigues dos Santos, K. P., Vieira, L. C., Lima, V. M. de, Coutinho, E. N., Carvalho, D. F. de, \& Ferreira, C. P. (2020). Qualidade de vida e prevalência de dor na região cervical em acadêmicos. Revista Eletrônica Acervo Saúde, 39, 1-9. https://doi.org/10.25248/reas.e2021.2020

Delfino, P. D.; Rampim, D. B.; Alfieri, F.; Tomikawa, L. C. O.; Fadel, G.; Stump, P. R. N. A. G.; Imamura, S. T.; Imamura, M.; Battistella, L. R.; Bernardo, W. M \& Andrada, N. C. Cervicalgia: reabilitação. Revista Acta Fisiátrica, 19(2), 73-81.

Fillingim, R. B., King, C. D., Ribeiro-Dasilva, M. C., Rahim-Williams, B., \& Riley, J. L., 3rd (2009). Sex, gender, and pain: a review of recent clinical and experimental findings. The journal of pain, 10(5), 447-485. https://doi.org/10.1016/j.jpain.2008.12.001

Gharib, N.M., \& Hamid, N. (2013). Prevalence of mechanical neck pain in Taif university female students: a survey study. Journal of American Science. v.9, n.6, p. 347-54.

Jenkins, M., Menéndez, C. C., Amick, B. C., Tullar, J., Hupert, N., Robertson, M. M., \& Katz, J. N. (2007). Undergraduate college students' upper extremity symptoms and functional limitations related to computer use: a replication study. Revista Work (Reading, Mass.). 28(3): 231-238.

Kanchanomai, S., Janwantanakul, P., Pensri, P., \& Jiamjarasrangsi, W. (2011). Risk factors for the onset and persistence of neck pain in undergraduate students: 1-year prospective cohort study. Revista BMC Public Health. 11: 2-8. https://doi.org/10.1186/1471-2458-11-566

Kim, H. J., DH, \& Kim, J. S. (2015). The relationship between smartphone use and subjective musculoskeletal symptoms and university students. Journal of physical therapy Science. 27(3), 575-579. https://doi.org/10.1589/jpts.27.575 
Kuć, J., \& Żendzian-Piotrowska, M. (2020). A Pilot Study Evaluating the Prevalence of Cervical Spine Dysfunction Among Students of Dentistry at the Medical University. Journal Frontiers in neurology. 11, 200. https://doi.org/10.3389/fneur.2020.00200

Lee, J., \& Seo, K. (2014). The comparison of cervical repositioning errors according to smartphone addiction grades. Journal of physical therapy Science. 26(4), 595-598. https://doi.org/10.1589/jpts.26.595

Mancin, G. B., Bonvicine, C., Gonçalves, C., Adriano, M., \& Barboza, I. (2008). Análise da influência do sedentarismo sobre a qualidade de vida de pacientes portadores de dor lombar crônica Analysis of sedentariness influence on the quality of life of chronic low back pain patients. Journal ConScientiae Saúde. 7(4):441-447

Mattos, G. D., Leite, C., Milman, L. d., Souza, L. D., Amaral, B., Lüdtke, I., \& Cabral, V. D. (2009). Cervicalgia em estudantes universitários do Sul do Brasil: prevalência e fatores associados. Revista Medicina de Reabilitação. 28, 15-20.

Mehrdad, R., Dennerlein, J. T., \& Morshedizadeh, M. (2012). Musculoskeletal disorders and ergonomic hazards among Iranian physicians. Archives of Iranian medicine. 15(6), 370-374.

Moher, D., Liberati, A., Tetzlaff, J., \& Altman, D. G. (2015). Principais itens para relatar Revisões sistemáticas e Meta-análises: A recomendação PRISMA. Revista de Epidemiologia e Serviços de Saúde, 24(2), 335-342. https://doi.org/10.5123/S1679-49742015000200017

Namwongsa, S., Puntumetakul, R., Neubert, M. S., \& Boucaut, R. (2018). Factors associated with neck disorders among university student smartphone users. Journal Work (Reading, Mass.). 61(3), 367-378. https://doi.org/10.3233/WOR-182819

Neto, A. de C. e S., Pereira, B. P. M., Sousa, J. de S. e, França, G. L. M. de, Fernandes, R. J., \& Gouveia, N. M. de. (2021). Alterações posturais da coluna cervical e cervicalgia associadas ao perfil dos acadêmicos de medicina: uma revisão integrativa/ Postural changes in the cervical column and cercivalgia associated with the profile of medicine academics: an integrative review. Brazilian Journal of Development. 7(3), 25540-25555. https://doi.org/10.34117/bjdv7n3-316

Oksanen, A. M., Laimi, K., Löyttyniemi, E., \& Kunttu, K. (2014). Trends of weekly musculoskeletal pain from 2000 to 2012: National study of Finnish university students. European Journal of Pain. 18(9): 1315-1622. https://doi.org/10.1002/j.1532-2149.2014.492.x

Oliveira, T. N. de, Ferreira, L. G. de F., Ferreira, L. M. de S., Gonçalves, M. R., Chaves, J. C., Silva, N. R. da, Pereira, K. J., Silva, P. R. C. da, Sousa, J. V. de, Silva, J. C. da, Silva, L. D. da, \& Dantas, E. P. de V. (2021). Prevalência de cervicalgia em estudantes de fisioterapia usuários de smartphones. In Fisioterapia e Terapia Ocupacional: Promoção \& Prevenção e Reabilitação 2. Atena Editora. https://doi.org/10.22533/at.ed.0212105015

Safiri, S., Kolahi, A. A., Hoy, D., Buchbinder, R., Mansournia, M. A., Bettampadi, D., Ashrafi-Asgarabad, A., Almasi-Hashiani, A., Smith, E., Sepidarkish, M., Cross, M., Qorbani, M., Moradi-Lakeh, M., Woolf, A. D., March, L., Collins, G.; Ferreira, M. L. (2020). Global, regional, and national burden of neck pain in the general population, 1990-2017: Systematic analysis of the Global Burden of Disease Study. 2017. V: 368: m791. 1-2

Sato, M. I., Aguiar, L. F., Freitas, M. N. V. de, Guerra, I., \& Martinez, J. E. (2019). Cervicalgia entre estudantes de medicina: uma realidade multifatorial. Revista Da Faculdade de Ciências Médicas de Sorocaba. 21(2), 55-58. https://doi.org/10.23925/1984-4840.2019v21i2a3

Silva, A. de F., Lima da Costa, M. A., Rodrigues Soutinho, R. R. S. S., \& Pedrosa, A. de S. (2017). Prevalência de Cervicalgia em Acadêmicos de Odontologia de um Centro Universitário. Revista Portal: Saúde E Sociedade. 2(2), 422-434. https://doi.org/10.28998/rpss.v2i2.3171

Silva, R. M. V. da, Lima, M. S. de, Costa, F. H., \& Silva, A. C. da. (2012). Efeitos da quiropraxia em pacientes com cervicalgia: revisão sistemática. Revista Dor. 13(1): 71-74

Smith, D. R., \& Leggat, P. A. (2004). Musculoskeletal disorders among rural Australian nursing students. Australian Journal of Rural Health. 12(6): 241-245. https://doi.org/10.1111/j.1440-1854.2004.00620.x

Teixeira, J.M., Barros Filho, T., Lin, T.Y., Hamani, C., Teixeira, W.G.J. (2001). Cervicalgias. Revista. Med. 80(2):307- 316

Tourinho, C. R. R. P., \& Santana Junior, V. (2020). Cervicalgia Inespecífica em Estudantes de Fisioterapia de uma Instituição Privada / Nonspecific Cervicalgia in Physiotherapy Students from a Private Institution. ID on Line. Revista de Psicologia, 14(53), 535-542. https://doi.org/10.14295/idonline.v14i53.2877

Vey, A. P. Z., Da Silva, A. C., \& De Lima, F. S. T. (2013). Análise de dor nas costas em estudantes de graduação. Disciplinarum Scientia. 14(2), 217-225

Vitta, A., Bento, T., Perrucini, P. O., Felippe, L. A., Poli-Frederico, R. C., \& Borghi, S. M. (2021). Neck pain and associated factors in a sample of high school students in the city of Bauru, São Paulo, Brazil: cross-sectional study. Revista paulista de medicina. 139(1), 38-45. https://doi.org/10.1590/15163180.2020.0168.R1.30102020

Woo, E., White, P., \& Lai, C. (2016). Musculoskeletal impact of the use of various types of electronic devices on university students in Hong Kong: An evaluation by means of self-reported questionnaire. Journal Manual therapy. 26, 47-53. https://doi.org/10.1016/j.math.2016.07.004 